\title{
Análisis de la generación de GEI en la cuenca del río Bogotá (Colombia) y un panorama comparativo entre las cuencas de las capitales de México y Perú
}

\author{
Analysis of GHG Generation in the Bogotá River Basin (Colombia) and Comparative \\ Panorama with the Basins of the Capital Cities of Mexico and Peru
}

\section{Diana Lucía Cristancho Montenegro ab , Jessica Estefanía Rojas Bernala , Juliana Bohórquez Mesa ${ }^{a}$ y María Fernanda Dueñas Escuderoac}

\footnotetext{
${ }^{a}$ Facultad de Ingeniería y Ciencias Básicas, Universidad Central, Colombia

${ }^{b}$ inge.dianacristancho26@gmail.com https://orcid.org/0000-0001-7417-2583

' https://orcid.org/0000-0001-9917-2504
}

\section{RESUMEN}

Este trabajo consiste en una revisión sistemática sobre el impacto de las actividades económicas, las plantas de tratamiento de aguas residuales (PTAR) y los factores climáticos (temperatura y precipitación) en la emisión de gases efecto invernadero (GEI), enfocándose primero en la cuenca del río Bogotá y posteriormente realizando un análisis comparativo con las cuencas del Valle de México (México) y el río Rímac (Perú). Debido a que la principal contaminación de las cuencas está dada por descargas de aguas residuales, se han implementado las PTAR para su mitigación. En esta investigación se presenta, el diagnóstico del estado actual de las (PTAR), y un análisis de los factores climatológicos y meteorológicos, además de las posibles afectaciones a nivel salud, aplicando la metodología del IPCC de 2006. En el caso de la cuenca del río Bogotá, la mayor generación de GEI corresponde a $\mathrm{CH} 4(82,163 \%)$ y $\mathrm{N} 2 \mathrm{O}(76,059 \%)$, sien-

Citation: Cristancho-Montenegro, D. L., Rojas-Bernal, J. E., BohórquezMesa, J. y Dueñas-Escudero, M. F. (2020). Análisis de la generación de GEl en la cuenca del río Bogotá (Colombia) y un panorama comparativo entre las cuencas de las capitales de México y Perú. Mutis, 10(2), 25-36. https://doi. org/10.21789/22561498.1674

Recibido: Febrero 26, 2020. Aceptado: Junio 15, 2020.

Copyright: @2020. Cristancho-Montenegro, D. L., Rojas-Bernal, J. E., Bohórquez-Mesa, J. y Dueñas-Escudero, M. F. (2020). This is an open-access article, which permits unrestricted use, distributions and reproduction in any medium, provided the original author and source are credited.

Competing Interests: The authors have no conflict of interest. do la cuenca media la que reporta mayores impactos, así: tratamiento mixto $(45,1 \%)$ con mayor influencia del cambio climático, actividades económicas industriales con alto impacto $(70,17 \%)$, factores climáticos como bajas temperaturas y precipitación media. De las cuencas del Valle de México, la cuenca baja reporta mayor impacto, así: tratamiento aerobio $(62,11 \%)$, actividades económicas comerciales $(56,10 \%)$, factores climáticos con una preferencia de temperatura y precipitaciones altas. De la cuenca del río Rímac, en Perú, la cuenca media reporta mayor impacto, así: tratamiento mixto $(49,4 \%)$, actividades económicas 
comerciales $(59,2 \%)$ y factores climáticos como precipitaciones y temperaturas altas. La validación de los datos anteriores fue confirmada con el inventario nacional de GEI reportado por cada país, evidenciando que el tratamiento, el tipo de actividad económica y el clima son factores que influyen en la generación de GEI. Además, se encontró que las enfermedades cardiovasculares son las afectaciones más recurrentes ocasionadas por la emisión de GEI.

Palabras clave: GEI, cuenca, tratamiento de aguas residuales, actividades económicas.

\section{ABSTRACT}

This study consists of a systematic review on the impacts generated by economic activities, wastewater treatment plants (WWTPS) processes, and climatic factors (temperature and precipitation) on greenhouse gases (GHG) emissions, focusing first on the Bogotá River Basin and, subsequently, conducting a comparative analysis with Valle de México (Mexico) and Rímac River (Peru) basins. Since the main pollution source of the basins is wastewater discharges, WWTPS have been implemented for mitigation. This research presents the diagnosis of the current state of WWTPS, an analysis of climatological and meteorological factors, in addition to the possible health effects, applying the 2006 IPCC methodology. In the case of the Bogotá River Basin, the largest generation of GHGS comes from $\mathrm{CH} 4$ (82.163\%) and $\mathrm{N} 2 \mathrm{O}$ (76.059\%), being its middle basin the most affected, according to results: mixed treatment (45.1\%) with great influence on climate change; economic industrial activities with high impact (70.17\%); and climatic factors such as low temperatures and average rainfall generating high concentration of GHG. In regard to the Valley of Mexico basins, the lower basin shows greater impact, as follows: aerobic treatment (62.11\%), economic trading activities (56.10\%), and high temperatures and rainfall. From the Rímac River Basin, in Peru, the middle basin reports greater impact, as follows: mixed treatment (49.4\%), economic trading activities (59.2\%), and climatic factors such as rainfall and high temperatures. The validation of the previous data was confirmed via the national GHG inventory reported by each country, showing that the treatment used, the type of economic activity and climate are variables that affect GHG emissions. It was also found that cardiovascular diseases are the most recurrent health issues caused by GHG emissions.

Keywords: GHG, basin, wastewater treatment, economic activities.

\section{INTRODUCCIÓN}

Actualmente, la calidad del recurso hídrico es un factor de gran importancia para la conservación de los ecosistemas y el desarrollo de la vida, en especial la vida humana. Las actividades antropológicas necesarias para el desarrollo de la humanidad y un sin número de necesidades relacionadas han generado una gran problemática ambiental respecto a la contaminación de los cuerpos de agua receptores y la calidad del aire, situación que afecta de alguna manera la calidad de los ecosistemas y la vida de las comunidades.

Según la Superintendencia Nacional de Servicios de Saneamiento de Perú:

La descarga directa sin tratamiento previo de las aguas residuales en los cuerpos receptores es uno de los principales factores de contaminación, no solo de los diversos ecosistemas existentes sino de las actuales fuentes de agua, lo que amenaza la sostenibilidad del recurso y pone en riesgo la salud de la población. Sumando a la enorme cantidad de gases de invernadero que se emiten a la atmósfera, y el problema que esto supone para el medio ambiente, se hace necesario tomar medidas para su reducción (Superservicios, 2017).

La mayoría de las cuencas a nivel mundial presentan condiciones desfavorables en la calidad del agua, por lo tanto, como parte de un intento por mejorar la calidad del agua y su conservación, se han implementado planes de saneamiento como las plantas de tratamiento de aguas residuales (PTAR), las cuales se encuentran distribuidas en estas (DNP, 2002). Así mismo, el aumento en las emisiones de gases de efecto invernadero (GEI) despierta un creciente interés en el sector del agua debido al impacto del cambio climático sobre la disponibilidad del recurso hídrico en algunas áreas (Barbu et al., 2017; Blanco et al. 2014; Delga- 
do et al., 2014). En cuanto a los procesos en las PTAR, Lizarazo-Becerra y Orjuela-Gutiérrez (2013, p. 19) afirman que:

La temperatura del agua residual es mayor que la del agua potable, esta varía entre 10 y 20 ㄷ C debido a que se añade calor al agua en los sistemas de plomería de las edificaciones, lo que genera gran cantidad de gases a la atmósfera.

De acuerdo con investigaciones relacionadas y estudios de impacto ambiental, entre otras investigaciones, autores como Parra et al. (2010, p. 87 ) afirman que: "En general, las PTAR producen la mayor cantidad de gases de $\mathrm{CH} 4$ y $\mathrm{CO} 2$, las cuales corresponden a las fuentes más importantes de emisión de GEI". A partir de lo anterior, surgen como preguntas de esta investigación los siguientes interrogantes: ¿qué tan influyentes son los factores climáticos y meteorológicos en el aumento de la concentración de GEI en las cuencas de estudio? y "¿qué tan significativas son las actividades económicas y la densidad poblacional en las áreas donde más se generan GEI?

Se debe tener en cuenta que el proceso de tratamiento de aguas residuales genera emisiones de GEI que, en una u otra medida, influyen en el cambio climático y las condiciones ambientales. Por ello, es necesario realizar una revisión sistemática sobre el estado actual de las PTAR en las cuencas objeto de estudio y analizar su posible impacto ambiental en cuanto a la generación de GEI, respondiendo así al objetivo principal de la presente investigación que es: "Realizar un análisis multidimensional de los procesos de tratamiento de aguas residuales municipales aplicables en los países en desarrollo, considerando diversos factores climáticos". Mediante la actualización, localización y complemento del diagnóstico del estado actual de las PTAR en la cuenca del río Bogotá (Colombia), con base en la metodología propuesta por el Grupo Intergubernamental de Expertos sobre el Cambio Climático (IPCC, por sus siglas en inglés), análogamente se realizará un comparativo de las PTAR de la cuenca del Valle de México (México) y la cuenca del río Rímac (Perú). Además, se estudiará el impacto de algunas actividades económicas y los factores climáticos temperatura y precipitación sobre la emisión de GEI.

\section{MATERIALES Y MÉTODOS}

\section{Áreas de estudio}

La cuenca del río Bogotá se localiza en el departamento de Cundinamarca (Colombia) y tiene una superficie total de $5.89 \mathrm{~km}^{2}$, que corresponden a cerca del $32 \%$ del total de la superficie departamental (CAR, 2006; IDEAM \& PNUD, 2016). Esta cuenca cuenta con 63 PTAR (CAR, 2010).

De otro lado, según Rodríguez-Tapia y Morales-Novelo (2013) y el Instituto Nacional de Ecología y Cambio Climático de México (INECC, 2018), la cuenca del Valle de México se ubica dentro de tres entidades federativas (México, Hidalgo y Tlaxcala), considerando 85 municipios, y cuenta con una superficie de $9.738 \mathrm{~km}^{2}$. De acuerdo con cifras de la Secretaría del Medio Ambiente de la Ciudad de México, la cuenca registra un total de 227 PTAR (Sedema, 2015).

Por su parte, la cuenca del río Rímac se ubica en la vertiente del Pacífico, enmarcándose en las provincias de Lima, Huarochirí y Yauli, ocupando un área de $3.503,95 \mathrm{~km}^{2}$, de los cuales $2.302 \mathrm{~km}^{2}$ corresponden a la cuenca húmeda (Astorayme et al., 2016). Según el Observatorio del Agua Chillón Rímac Lurín (2017), la cuenca cuenta con 77 PTAR.

\section{Depuración y clasificación de la información re- colectada}

Con base en la información presentada en el apartado anterior, y siendo el objetivo de esta investigación, se identificó la localización de las PTAR a lo largo de las cuencas objeto de estudio, teniendo en cuenta la información disponible (estado actual, población, tipo de sistema, caudal de tratamiento y parámetros fisicoquímicos), como se observa en la figura 1.

De acuerdo con datos suministrados por los entes de control colombianos, se identificó que $45,1 \%$ de las PTAR realizan un tratamiento mixto de aguas, 39,2 \% ejecutan tratamiento aerobio y $15,6 \%$ tratamiento anaerobio. Según el Instituto de Hidrología, Meteorología y Estudios Ambientales de Colombia (IDEAM), la temperatura media anual de la cuenca se encuentra entre 12,8 y $27,8^{\circ} \mathrm{C}$ y su precipitación entre 599 y $1.700 \mathrm{~mm}$ anuales. Además, en esta habitan 1.297 .752 personas y se presenta un acumulado de actividades 


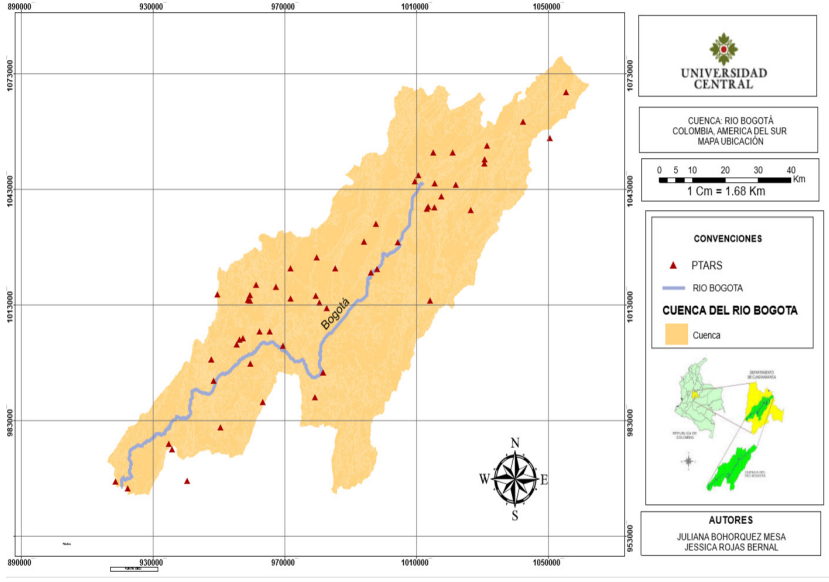

Figura 1. Inventario de PTAR en la cuenca del río Bogotá. Fuente: elaboración propia con base en USTA (2017) y CAR (2012).

económicas como industria $(70,17 \%)$, agricultura $(16,68 \%)$ y comercio $(13,15 \%)$, según datos de la Corporación Autónoma Regional (CAR) de Cundinamarca (2012), Vivid Economics (2015) y Gil-Gómez (2014).

Por otro lado, la figura 2 muestra las 227 PTAR existentes a lo largo de la cuenca del Valle de México, con base en información del compendio federal y la Comisión Nacional del Agua de México (Conagua). Del total de estas PTAR, $62,11 \%$ realiza tratamiento aerobio, $22,02 \%$ tratamiento mixto y $11,89 \%$ tratamiento anaerobio. La temperatura promedio más alta en la cuenca del Valle de México es de $24.5^{\circ} \mathrm{C}$ y la precipitación promedio más alta de $1.600 \mathrm{~mm}$. El acumulado de la actividad económica en esta región corresponde a comercio $(56,10 \%)$, agricultura $(26,07 \%)$ e industria $(17,82 \%)$ (Sedema, 2015; INECC, 2018).

En el caso del río Rímac, la figura 3 permite evidenciar la ubicación de 77 PTAR a lo largo de la cuenca de este cuerpo de agua. A partir de un diagnóstico realizado por la Sunass, se identifica que $49,4 \%$ de estas PTAR realiza tratamiento mixto, $42,8 \%$ tratamiento aerobio y el $7,8 \%$ restante ejecuta tratamiento anaerobio. La temperatura promedio en esta cuenca es de 14,24 a $19,42{ }^{\circ} \mathrm{C}$ y la precipitación promedio de $826,7 \mathrm{~mm}$. En cuanto al acumulado de actividades económicas, el comercio se ubica en el primer lugar $(59,2 \%)$, seguido de la agricultura $(22,4 \%$ ) y la industria $(14,3 \%)$, según cifras del Proyecto Segunda Comunicación Nacional de Cambio Climático (CMNUCC, 2009) y Calla-Llontop y Cabrera-Carranza (2010).

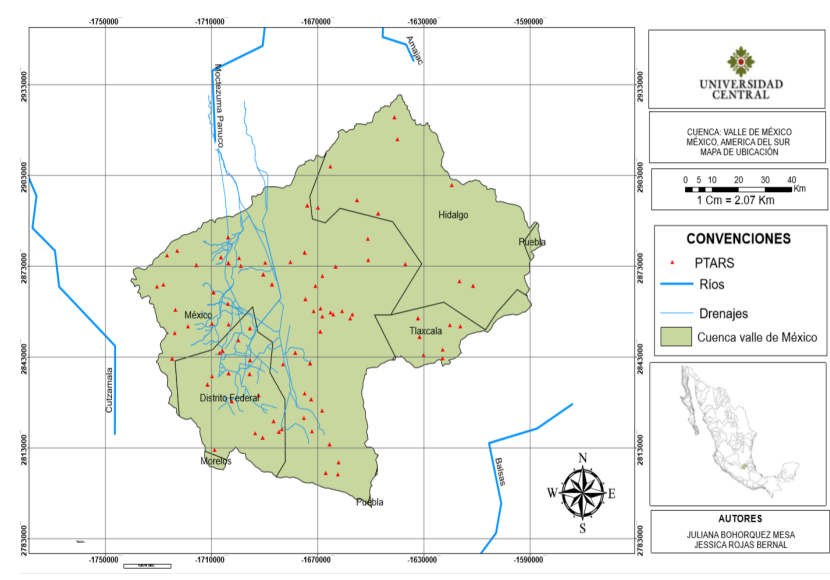

Figura 2. Inventario de PTAR en la cuenca de Valle de México. Fuente: elaboración propia con datos de Conagua (2015) e INECC (2018).

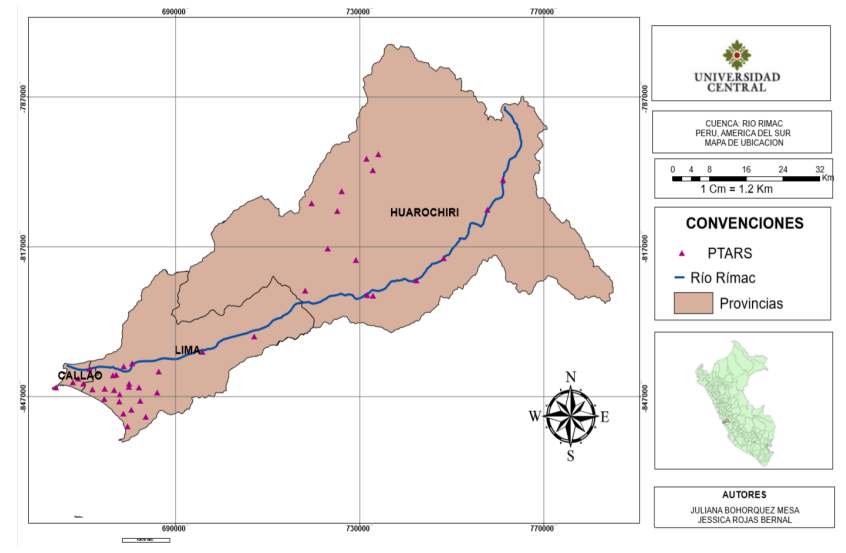

Figura 3. Inventario de PTAR en la cuenca del río Rímac.

Fuente: elaboración propia con base en Observatorio del Agua Chillón Rímac Lurín (2017) y Sunass (2017).

El análisis de los factores climatológicos y meteorológicos influyentes en los procesos desarrollados en las PTAR de las cuencas analizadas y las emisiones de GEI derivadas de ello se lleva a cabo teniendo en cuenta información suministrada por artículos de revisión y cifras de entidades meteorológicas con respecto al área de análisis. A partir del análisis de los factores climáticos precipitación y temperatura se identifican las emisiones de GEI que generan mayor impacto al ambiente.

Para la evaluación de la generación de GEl en las PTAR de Colombia se tomó como referencia el método propuesto por el IPCC en 2017. Para ello, se debe tener en cuenta que los procesos ejecutados por las PTAR pueden generar $\mathrm{CH} 4$ y N2O, los cuales tiene un potencial de calentamiento global 21 y 310 veces superior al del CO2, respectivamente. Según la meto- 
dología del IPCC (Grillenwater et al., 2008), el CO2 no debe considerarse en esta ecuación por ser un compuesto de origen biogénico, es decir, el carbono vuelve a la atmósfera de la misma forma en que hubiera ocurrido naturalmente (Nolasco, 2010). En el caso de las PTAR estudiadas en México y Perú no se consideraron las directrices del IPCC, sino que se tuvo en cuenta el inventario nacional de cada país. En una próxima fase de estudio deberá ejecutarse con mayor profundidad un análisis a la luz de dichas directrices, lo cual no hace parte del alcance del presente trabajo.

\section{RESULTADOS}

\section{Evaluación del número de PTAR y tipos de sistemas de tratamiento en cada cuenca}

\section{Colombia}

La figura 4 permite identificar que los municipios con mayores emisiones de GEl en la cuenca media del río Bogotá son Bogotá y Soacha, mientras que en su cuenca baja son Girardot y Granada, y en el caso de la cuenca alta Chía y Zipaquirá. Estos municipios corresponden a aquellos que registran un gran número de habitantes. En la figura 5 se relacionan las emisiones de GEI de acuerdo con el sistema de tratamiento empleado en las PTAR evaluadas en la cuenca del río Bogotá.

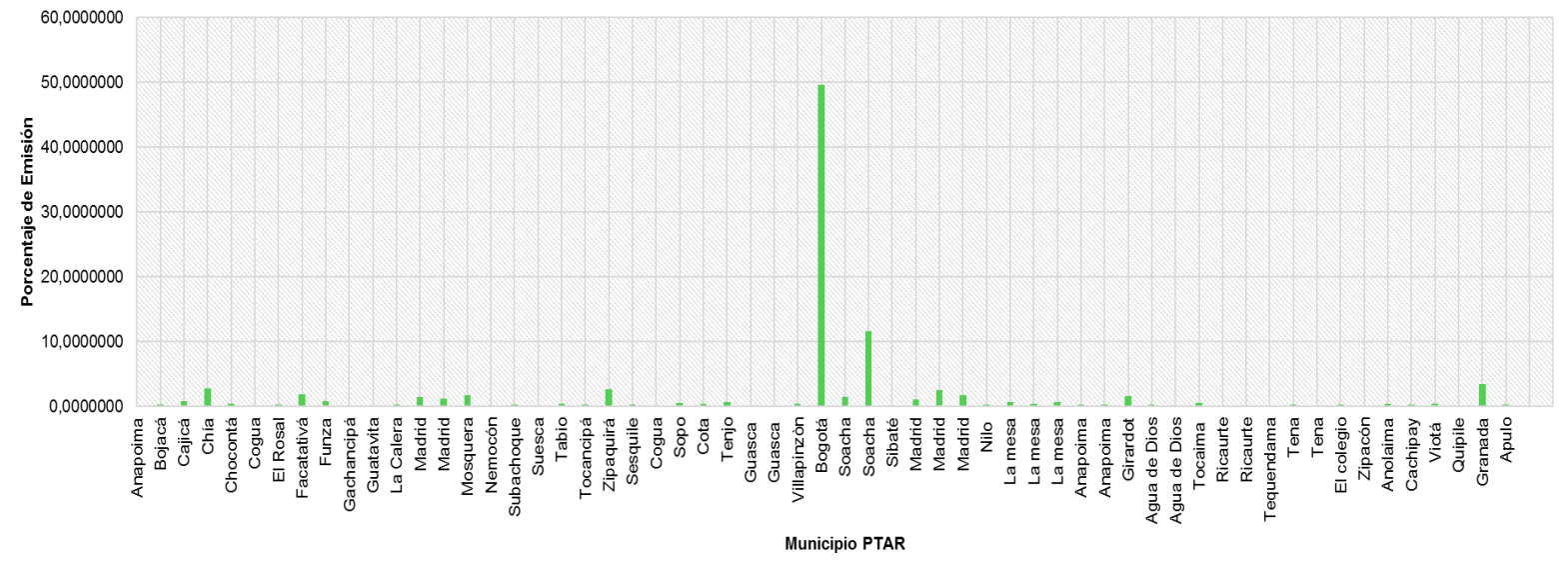

Figura 4. Resultados de GEI en la cuenca del río Bogotá. Fuente: elaboración propia con base en CAR (2018).

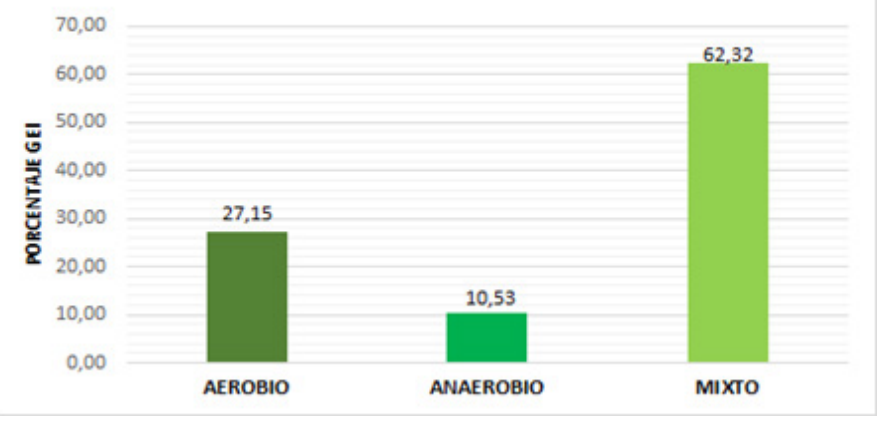

Figura 5. Resultados de porcentaje de GEI.

Fuente: elaboración propia con base en Ashrafi et al. (2013), CAR (2018), IDEAM y PNUD (2016), Meneses y Hernández (2004) y Pan et al. (2011). 
Con respecto al porcentaje de emisiones de GEI en las PTAR analizadas según el sistema de tratamiento implementado en cada municipio, se obtuvo que las plantas con tratamiento mixto registran mayores emisiones de GEI (62,32\%), siendo el tipo de tratamiento más utilizado con tecnología de lagunas de estabilización. Por su parte, las plantas con tratamiento anaerobio son las que menos emisiones generan $(10,53 \%)$, con lagunas UASB. Por último, en el tratamiento aerobio la tecnología predominante son los sistema de aireadores. La figura 6 muestra la relación de GEI con respecto al tipo de tratamiento de aguas residuales.

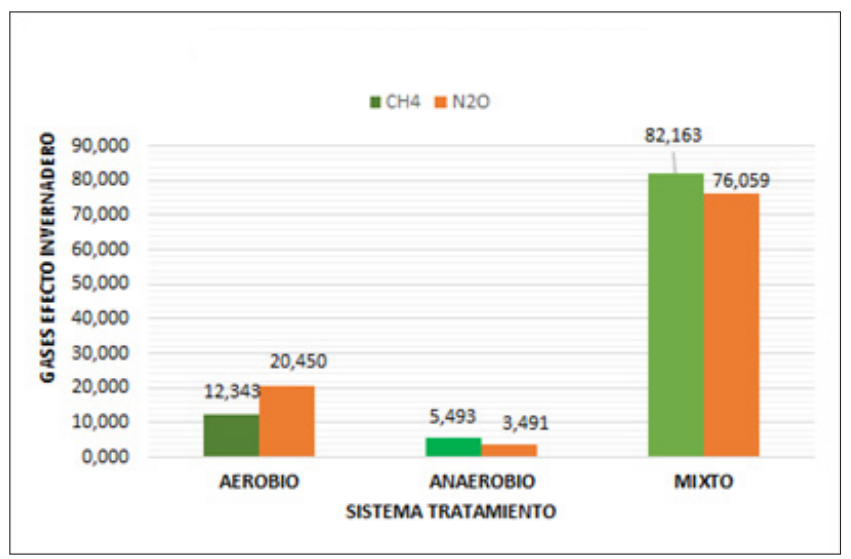

Figura 6. Resultados de GEI según el sistema de tratamiento empleado Fuente: elaboración propia con base en CAR (2018), EPA (2010), El-Fadel y Massoud (2001), Kyung et al. (2015), Molinos-Senante y Guzmán (2018) y Mouri et al. (2013).

Teniendo en cuenta que, bajo ciertas condiciones, durante el tratamiento biológico de aguas residuales se genera metano y óxido nitroso, la figura 5 permite identificar que el GEI de mayor emisión en el sistema de tratamiento anaerobio y el sistema mixto es el metano ( 5,493 y $82,163 \%$, respectivamente). Esto puede deberse a que un proceso anaeróbico no requiere suministro de oxígeno (con un consumo energético mucho menor) y genera una cantidad de lodos considerablemente menor. Así mismo, este proceso permite la obtención de metano como producto de la degradación de la materia orgánica, el cual puede ser utilizado con fines energéticos. Por otro lado, se evidencia que el sistema aerobio presenta altos niveles de NO2 (20,450 \%).

\section{Análisis de dispersión de GEI en la cuenca del río Bogotá}

A continuación se presenta el mapa de dispersión de GEI en la cuenca del río Bogotá a partir de las emisiones de las PTAR objeto de estudio (figura 7).

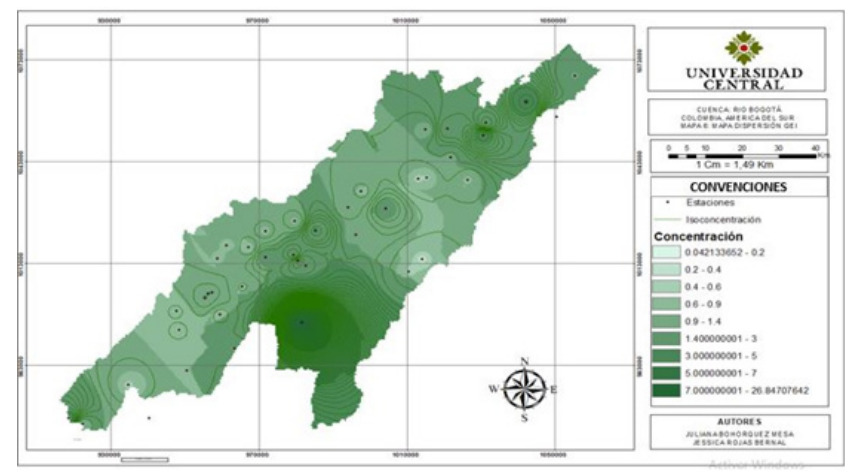

Figura 7. Mapa de dispersión de GEI en la cuenca del río Bogotá Fuente: elaboración propia con base en CAR (2018).

Como se aprecia en la figura 7, la contaminación por GEI es mayor en las cuencas media y alta, lo cual se debe a que la principal zona industrial, ganadera y agrícola del departamento de Cundinamarca se encuentra ubicada en estas dos cuencas. Adicionalmente, la población de los municipios aledaños tiende a crecer rápidamente, lo cual afecta a las PTAR de la región, puesto que a mayor concentración de población mayor es el volumen de materia orgánica que debe ser tratado y, por ende, mayor generación y emisiones de GEI.

\section{México}

En la cuenca del Valle de México existen 227 PTAR, 141 con tratamiento aerobio $(62,11 \%), 50$ con tratamiento mixto $(22,02 \%)$ y 27 con tratamiento anaerobio (11,89 \%) (Conagua, 2010; Magallón-Andalón, 2007), de manera que los sistemas de tratamiento más utilizados son lodos activados, las lagunas de estabilización y RAFA o WASB, como se muestra en la figura 8.

\section{Inventario nacional de emisión de GEI}

Según cifras del INECC (2015), el 71,11 \% de las emisiones de GEI en México resultan de la producción de energía, 7,74 \% de procesos industriales, 14,59\% de actividades agrícolas, silvicultura y uso del suelo, y $5 \%$ son ocasionadas por la generación de residuos. 


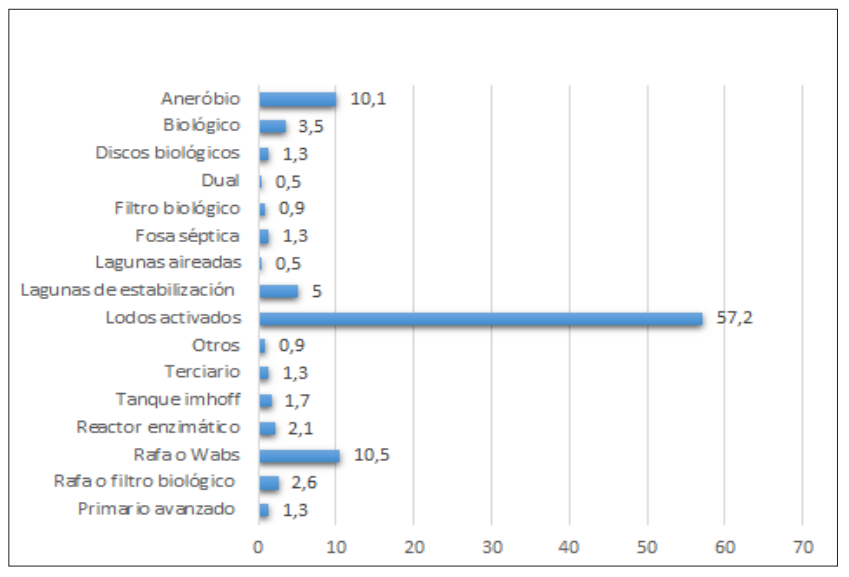

Figura 8. Númrto de PTAR y tipo de proceso empleado en México Fuente: elaboración propia con base en Conagua (2010).

Esta última se divide en dos categorías: (i) tratamiento y eliminación de aguas residuales (49\%) y (ii) eliminación de residuos y otros (51\%). Teniendo en cuenta esta última actividad, la emisión total de GEI por el tratamiento y eliminación de aguas residuales en el inventario nacional corresponde a 3,21 \% del total de emisiones.

\section{Perú}

En el caso de Perú, la tecnología de tratamiento secundario más empleada son las lagunas, bien sea anaerobias, facultativas o aireadas, las cuales están presentes en $75 \%$ de las PTAR de este país, de manera individual o combinada. También se cuenta con tecnología de lodos activados en las variedades de flujo continuo y SBR, lechos fijos sumergidos, filtros percoladores y reactores anaerobios tipo RAFA (Sunass, 2017), como se evidencia en la figura 9.

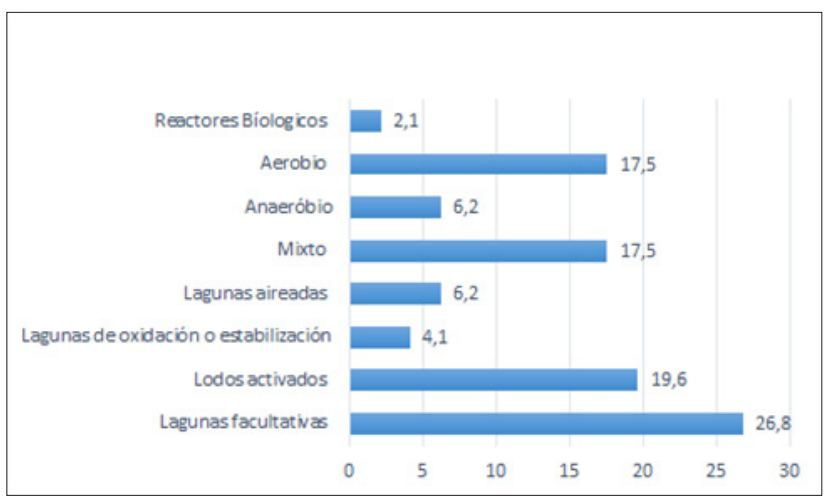

Figura 9. Tipo de sistema y tecnología empleados en las PTAR de Perú Fuente: elaboración propia con base en Sunass (2015).

\section{Inventario nacional de emisión de GEI}

En relación con las actividades económicas, según el Ministerio de Ambiente de Perú (2016), el país reporta un $26 \%$ de emisiones a causa de la producción de energía, $3 \%$ por procesos industriales, $66 \%$ por actividades de agricultura, silvicultura y uso del suelo y $5 \%$ por generación residuos. Esta última variable se divide en dos categorías: (i) tratamiento y eliminación de aguas residuales $(15,80 \%)$ y (ii) eliminación de residuos y otros $(84,20 \%)$. Teniendo en cuenta estas cifras, las emisiones totales de GEI ocasionadas por el tratamiento y eliminación de aguas residuales en el inventario nacional es de 0,79\%.

\section{Evaluación de los factores climáticos de las cuen- cas estudiadas}

Los factores climáticos y meteorológicos influyen directamente en la calidad del agua, la emisión de GEI y el comportamiento de las PTAR, puesto que las condiciones de alta precipitación y altas temperaturas ocasionan una mayor generación de GEI, siendo la cuenca media del río Bogotá, la cuenca baja del Valle de México y la cuenca media del río Rímac las más afectadas por este fenómeno entre el grupo de cuencas analizadas (figura 10). Las PTAR en estas cuencas, en su mayoría, llevan a cabo tratamiento mixto, por lo cual los gases no logran dispersarse totalmente en la atmósfera.

\section{Análisis de GEI en relación con las actividades económicas de los países evaluados}

Dentro de la evaluación realizada de las actividades e industrias que se encuentran en las cuencas principales de Ciudad de México (México), Lima (Perú) y Bogotá (Colombia), se logró identificar la influencia de estas actividades en la emisión de GEI, (tabla 1).

En la tabla 1 se reflejan las actividades económicas más significativas de Colombia, México y Perú, observando que las actividades con mayor generación de GEI son la industria $(61,82 \%)$, el comercio $(20,54 \%)$ y la agricultura $(16,26 \%)$, realizando un promedio. Además, se identificaron los diferentes tipos de GEI generados en cada país, con su respectivo porcentaje, como se observa en la tabla 2. 


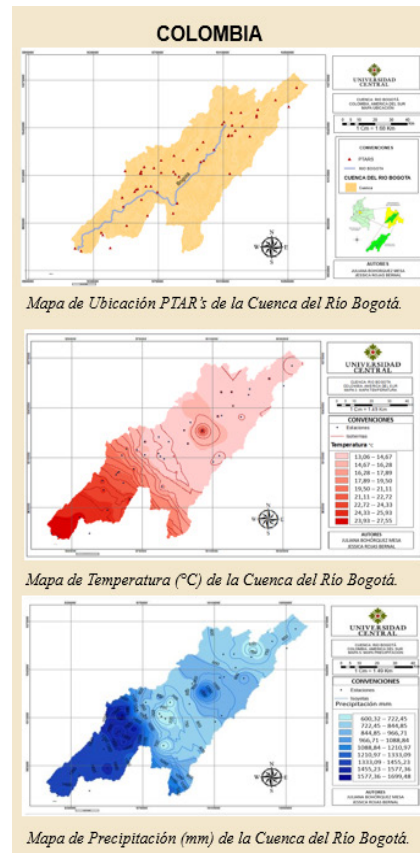

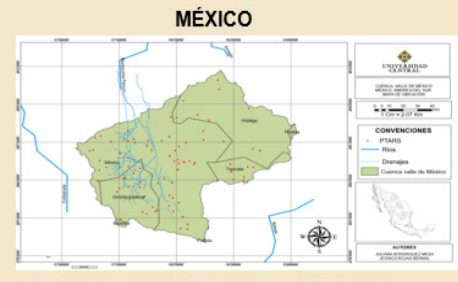

Mapa de Ubicación PTAR's de la Cuenca Valle de México.

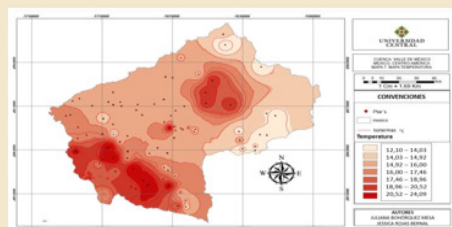

Mapa de Temperatura $\left({ }^{\circ} \mathrm{C}\right)$ de la Cuenca Valle de México.

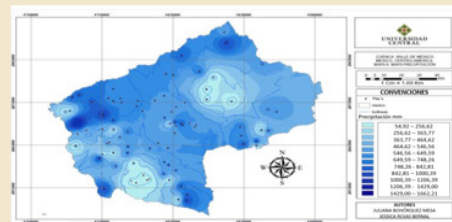

Mapa de Precipitación (mm) de la Cuenca Valle de México.

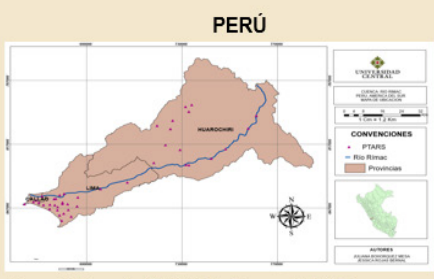

Mapa de Ubicación PLAR's de la Cuenca del Rio Rímac.

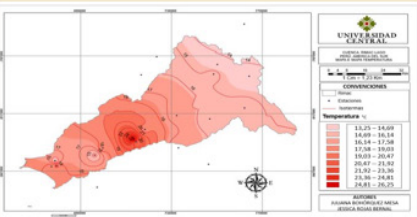

Mapa de Temperatura $\left({ }^{\circ} \mathrm{C}\right)$ de la Cuenca del Rio Rimac.

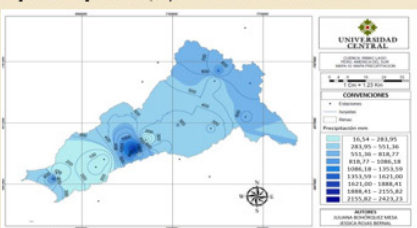

Mapa de Precipitación (mm) de la Cuenca del Rio Rimac.

Figura 10. Ubicación de las PTAR, temperatura y precipitación por cuencas

Fuente: elaboración propia con base en IDEAM y PNUD (2016), INECC (2018) y MAR y ANA (2016).

Tabla 1. Actividades económicas desarrolladas en los países evaluados

\begin{tabular}{|c|c|c|}
\hline $\begin{array}{c}\text { Colombia } \\
\text { (Cuenca río Bogotá) }\end{array}$ & $\begin{array}{c}\text { México } \\
\text { Perú } \\
\text { (Cuenca río Rímac) }\end{array}$ \\
\hline Industria $(70,17 \%)$ & Industria $(56,10 \%)$ & Industria $(59,2 \%)$ \\
Agricultura $(16,68 \%)$ & Agricultura $(17,82 \%)$ & Agricultura $(14,3 \%)$ \\
Comercio $(13,15 \%)$ & Comercio $(26,07 \%)$ & Comercio $(22,4 \%)$ \\
\hline
\end{tabular}

Fuente: elaboración propia con base en MinAmbiente (2016), Conagua (2015), CAR (2016) y Fernández-Estela (2011).

Tabla 2. Tipos de GEI generados por las actividades económicas en los países evaluados.

\begin{tabular}{|c|c|c|}
\hline $\begin{array}{c}\text { Colombia } \\
\text { (Cuenca Río Bogotá) }\end{array}$ & $\begin{array}{c}\text { México } \\
\text { (Cuenca Valle de México) }\end{array}$ & $\begin{array}{c}\text { Perú } \\
\text { (Cuenca río Rímac) }\end{array}$ \\
\hline $\begin{array}{l}\text { Ozono, aerosoles ácidos, material particulado } \\
\text { y monóxido de carbono afectados por la } \\
\text { temperatura, humedad relativa y luz solar, metano } \\
\text { y óxido nitroso. } \\
\text { Colombia emite 3,66 t CO2 eq. per cápita/año, con } \\
\text { el } 62,6 \% \text { de las emisiones del país. }\end{array}$ & $\begin{array}{l}\text { Monóxido de carbono (CO: } 33 \text { mill ton; } 70 \% \text { del } \\
\text { total), compuestos orgánicos volátiles (COV: 6,03 } \\
\text { mill t; } 13 \% \text { ), óxido de nitrógeno (NOX: 3,2 mill t; } \\
7 \% \text { ) y bióxido de azufre (SO2: } 2,2 \text { mill t; } 5 \% \text { ). El } \\
\text { resto de los contaminantes poseen un porcentaje } \\
\text { igual o menor a } 2 \% \text {. }\end{array}$ & $\begin{array}{l}\text { Emisión de } 171.310 \text { giga gramos de dióxido de } \\
\text { carbono, metano, óxidos de nitrógeno. De los } \\
\text { cuales } 35 \% \text { corresponde a la tala indiscriminada y } \\
20 \% \text { a transporte vehicular y generación eléctrica. }\end{array}$ \\
\hline
\end{tabular}

Fuente: elaboración propia con base en U. Loyola Perú (2014) y oms (2018).

A partir de la información en la tabla 2 , se deduce que en los tres países los GEI que más se generan son dióxido de carbono (CO2), metano ( $\mathrm{CH} 4)$, óxido nitroso (N2O) y clorofluorocarbonos (CFC). En cuan- to a Colombia, según el IDEAM (2015), el CO2 proviene del sector forestal y el $\mathrm{CH} 4$ del sector agropecuario. En cuanto a México, según el Instituto Nacional de Estadística y Geografía (INEGI, 2010), 
el porcentaje de $\mathrm{CO} 2$ se presenta por la quema de combustibles fósiles y los procesos industriales. Por su parte, las emisiones de $\mathrm{CH} 4$ corresponden a la categoría de desechos, energía y agricultura, y las de N2O al manejo de excretas y el uso de fertilizantes nitrogenados. En cuanto a Perú, según MinAmbiente (2016), se evidencia que el mayor porcentaje de $\mathrm{CO} 2$ se presenta por el uso y cambio del suelo, la silvicultura y la emisión de combustibles en el sector del transporte.

\section{Análisis de posibles afectaciones a nivel salud por la generación de GEI}

La tabla 3 da cuenta de las posibles afectaciones a nivel de salud más representativas en México, Perú y Colombia que son ocasionadas por la emisión de GEI. A partir de esta información, se puede observar que en los tres países las afectaciones más comunes son accidentes cardio vasculares (42,2\%), enfermedades pulmonares (14,9\%) y cáncer de pulmón (8,3\%).

Tabla 3. Listado de posibles afectaciones a nivel salud resultado de GEI

\begin{tabular}{|c|c|c|c|}
\hline & Colombia (C. río Bogotá) & México (C. Valle de México) & Perú (C. río Rímac) \\
\hline Enfermedades & $\begin{array}{c}\text { Dificultad respiratoria, dolor de cabeza, } \\
\text { malestar en ojos, nariz y pulmones, el } \\
58 \% \text { se debe a cardiopatías isquémicas } \\
\text { y accidentes cerebrovasculares, el } 18 \% \\
\text { a enfermedad pulmonar obstructiva } \\
\text { crónica e inspecciones respiratorias } \\
\text { agudas y el } 6 \% \text { se debe al cáncer de } \\
\text { pulmón. }\end{array}$ & $\begin{array}{l}\text { La contaminación del aire fue responsable } \\
\text { de 3,7 millones de muertes en el planeta } \\
\text { (11\% por enfermedad pulmonar obstructiva } \\
\text { crónica, el } 6 \% \text { por cáncer de pulmón, } 40 \% \\
\text { por enfermedad isquémica del corazón, } 40 \% \\
\text { por accidentes cerebrovasculares y el 3\% por } \\
\text { infección respiratoria aguda). }\end{array}$ & $\begin{array}{l}20,8 \% \text { infecciones respiratorias agudas, } \\
\text { cáncer de piel, asmas y alergias. } 11,8 \% \\
\text { tracto respiratorio agudo, } 15,8 \% \\
\text { enfermedad pulmonar obstructiva } \\
\text { crónica, } 13 \% \text { cáncer de pulmón, } 10 \% \\
\text { enfermedad cardiaca isquémica y } 28,6 \% \\
\text { accidentes cerebrovasculares. }\end{array}$ \\
\hline
\end{tabular}

Fuente: elaboración propia con base en ULoyola Perú, (2014) INS, (2009) y OMS (2018).

\section{DISCUSIÓN Y CONCLUSIONES}

Para la cuenca del río Bogotá (Colombia) se encontró que los registros de precipitación y temperaturas son altos, presentándose una mayor incidencia en la generación de GEI. Además, se identifica que la actividad industrial genera la mayor proporción de emisiones de $\mathrm{CH} 4$ y $\mathrm{N} 2 \mathrm{O}$ (70,17\%). El CH4 es el gas que genera mayor impacto ambiental en la cuenca media debido a los asentamientos humanos propios de la cercanía a la capital del país, el número de PTAR en operación y las actividades económicas mencionadas. El análisis de dispersión muestra que la concentración de GEI se encuentra en la cuenca media, lo cual se debe, en parte, a las precipitaciones y temperaturas medias observadas. No obstante, prevalece la influencia de las actividades económicas y la densidad poblacional en la concentración de este tipo de gases.

En la cuenca del Valle de México (México) se encontró que el proceso más utilizado en las PTAR es el aerobio con lodos activados. En la cuenca baja se registran altas temperaturas y altas precipitaciones, focalizadas en diferentes puntos de la cuenca. Así mismo, se re- porta una intensa actividad económica (industria y comercio) y una alta concentración de población debido a su proximidad a la capital del país, lo cual genera un alto impacto ambiental que puede generar mayor calentamiento, alterando así los recursos naturales y la calidad del aire.

En lo relacionado con la cuenca del río Rímac (Perú), se identificó que el proceso más utilizado en las PTAR es mixto con lagunas facultativas. En la cuenca media los registros de precipitación y temperaturas son los más altos, por lo que inciden en gran medida en la generación de GEI debido a que retardan el proceso de dispersión y liberación de emisiones en la atmósfera. Así mismo, el desarrollo de actividades económicas como el comercio, debido a la movilidad por intercambio comercial y los asentamientos humanos, es un factor que influye en la generación de GEI en esta región.

De acuerdo con los resultados de esta investigación, es posible deducir que la mayor concentración de GEI 
ocurre en la cuenca de estudio localizada en México, en la cual se observaron altas temperaturas y precipitaciones, además de un mayor número de población. Adicionalmente, este estudio logró identificar posibles afectaciones para la salud humana como consecuencia de las emisiones de GEI, entre las cuales los accidentes cardiovasculares son la patología más común.

\section{REFERENCIAS}

Ashrafi, O., Yerushalmi, L., \& Haghighat, F. (2013). Greenhouse gas emission by wastewater treatment plants of the pulp and paper industry. Modeling and simulation. International Journal of Greenhouse Gas Control, 17, 462-472. https://doi. org/10.1016/j.ijggc.2013.06.006

Astorayme, M. A., Gutiérrez, R. R., García, J., Felipe, O., \& Suarez, W. (2016). Downscaling temporal de un modelo hidrológico a paso diario adecuado en la cuenca del Rímac, Perú. XXVII Congreso Latinoamericano de Hidráulica, LADHI.

Barbu, M., Vilanova, R., Meneses, M., \& Santin, I. (2017). On the evaluation of the global impact of control strategies applied to wastewater treatment plants. Journal of Cleaner Production, 149, 396-405. https://doi.org/10.1016/j.jclepro.2017.02.018

Blanco, D., Collado, S., Díaz, M., Suárez, M. P., Pérez, J. A., \& Sánchez, J. (2014). Análisis de la huella de carbono en una planta de tratamiento de agua tipo carrusel. TECNOAQUA, 6, 52-57.

Calla-Llontop, H. C., \& Cabrera-Carranza , C. (2010). Calidad del agua en la cuenca del Río Rímac, sector de San Mateo, afectado por las actividades mineras. Revista del Instituto de Investigaciones FIGMMG, 13(25), 87-94. https://doi.org/10.15381/ iigeo.v13i25.399

Comisión Nacional del Agua de México [Conagua]. (2015). Inventario nacional de plantas municipales de potabilización y tratamiento de aguas residuales en operación. Conagua

Corporación Autónoma Regional de Cundinamarca [CAR] (2006). Plan de ordenación y manejo de la cuenca hidrográfica del río Bogotá. CAR.
Corporación Autónoma Regional de Cundinamarca [CAR] (2012). Plan de gestión ambiental regional PGAR 2012-2023. CAR.

Delgado, L., Poussade, Y., \& Aguiló, P. (2014). Huella de carbono de la regeneración de agua. https://www. aguasresiduales.info/revista/articulos/huella-decarbono-de-la-regeneracion-de-agua

Departamento Nacional de Planeación de Colombia [DNP]. (2002, 15 de julio). CONPES. http://www. minvivienda.gov.co/conpesagua/3177\%20-\%20 2002.pdf

El-Fadel, M., \& Massoud, M. (2001). Methane emissions from wastewater management. Environmental Pollution, 114, 177-185. https:// doi.org/10.1016/S0269-7491(00)00222-0

Environmental Protection Agency [EPA] (2010). Greenhouse gas emissions estimation methodologies for biogenic emissions from selected source categories. https://www.epa. gov/air-emissions-factors-and-quantification/ greenhouse-gas-emissions-estimationmethodologies-biogenic

Fernández-Estela, A. (2017). Aguas Residuales en el Perú, Problemática y Uso en la Agricultura. Lima: Autoridad Nacional del Agua Ministerio de Agricultura.

Gil-Gómez, J. A. (2014). Determinación de la calidad del agua mediante variables fisicoquímicas, y la comunidad de macroinvertebrados como bioindicadores de calidad del agua en la cuenca del río Garagoa (tesis de maestría, Universidad de Manizales). Repositorio Universidad de Manizales.

Grillenwater, M., Saarinen, K., \& Ajavon, A.-L. N. (2008). Precursors and Indirect Emissions. En S. Eggleston, L. Buendia, K. Miwa, T. Ngara \& K. Tanabe (eds.), 2006 IPCC Guidelines for National Greenhouse Gas Inventories (p. 16). IPCC.

Instituto de Hidrología, Meteorología y Estudios Ambientales [IDEAM] \& Programa de las Naciones Unidas para el Desarrollo [PNUD]. (2016). Inventario nacional y departamental de gases de efecto invernadero (GEI) Colombia. IDEAM \& PNUD. http://documentacion.ideam.gov.co/openbiblio/ bvirtual/023634/INGEI.pdf 
Instituto Nacional de Ecología y Cambio Climático de México [INECC]. (2018). Inventario nacional de emisiones de gases y compuestos de efecto invernadero 1990-2015. INECC.

Kyung, D., Kim, M., Chang, J., \& Lee, W. (2015). Estimation of greenhouse gas emissions from a hybrid wastewater treatment plant. Journal of Cleaner Production, 95, 117-123. https://doi. org/10.1016/j.jclepro.2015.02.032

Lizarazo-Becerra, J. M., \& Orjuela-Gutiérrez, M. I. (2013).Sistemas de plantas de tratamiento de aguas residuales en Colombia (tesis de especialización, Universidad Nacional de Colombia). Repositorio Institucional Universidad Nacional de Colombia.

Magallón-Andalón, M. (2007). Caracterización del lixiviado generado en el vertedero de Metepec $y$ análisis de medios reactivos permeables (tesis de maestría, Universidad Autónoma del Estado de México). Repositorio Institucional Universidad Autónoma del Estado de México.

Meneses, A., \& Hernández, E. E. (2004). Identificación de emisiones directas e indirectas de GEI en el sector tratamiento y disposición de aguas residuales: bases para la formulación de proyectos MDL En PTAR. BISTUA, 21(1), 60-69.

Ministerio de Agricultura y Riego de Perú (2010). Evaluación de los recursos hídricos en la cuenca del río Rímac. Ministerio de Agricultura y Riego de Perú.

Ministerio de Agricultura y Riego de Perú [MAR] \& Autoridad Nacional del Agua de Perú [ANA]. (2016). Priorización de cuencas para la gestión de los recursos hídricos. MAR \& ANA.

Ministerio de Ambiente de Perú [MinAmbiente]. (2016). Tercera comunicación nacional del Perú a la Convención Marco de las Naciones Unidas sobre el Cambio Climático. MinAmbiente.

Molinos-Senante, M., \& Guzmán, C. (2018). Reducing $\mathrm{CO} 2$ emissions from drinking water treatment plants: A shadow price approach. Applied Energy, 210, 623-631. https://doi.org/10.1016/j. apenergy.2016.09.065
Mouri, G., Takizawa, S., Fukushi, K., \& Oki, T. (2013). Estimation of the effects of chemically-enhanced treatment of urban sewage system based on life-cycle management. Sustainable Cities and Society, 9, 23-31. https://doi.org/10.1016/j. scs.2013.02.003

Nolasco, D. A. (2010). Desarrollo de proyectos MDL en Plantas de tratamiento de aguas residuales. Banco Interamericano de Desarrollo.

Observatorio del Agua Chillón Rímac Lurín. (2017). Análisis geoespacial de canales de riego en relación a las plantas de tratamiento de aguas residuales (PTAR) y áreas verdes en el ámbito de Lima metropolitana. Observatorio del Agua Chillón Rímac Lurín.

Organización Mundial de la Salud [OMS]. (2018). Cambio climático y sus efectos en la salud. oMs.

Pan, T., Zhu, X.-D., \& Ye, Y.-P. (2011). Estimate of life-cycle greenhouse gas emissions from a vertical subsurface flow constructed wetland and conventional wastewater treatment plants: A case study in China. Ecological Engineering, 37(2), 248-254. https://doi.org/10.1016/j. ecoleng.2010.11.014

Parra, R., Apaza, M., \& Agramont, A. (2010). Estimación de factores de emisión de gases de efecto invernadero en una planta de tratamiento de aguas residuales. Revista Boliviana de Química, $27(2), 81-88$.

Proyecto Segunda Comunicación Nacional de Cambio Climático (CMNUCC). (2009). Inventario nacional integrado de emisiones de gases efecto invernadero del Perú en el año 2000. MinAmbiente.

Rodríguez-Tapia, L., \& Morales-Novelo, J. A. (2013). Integración de un sistema de cuentas económicas e hídricas en la Cuenca del Valle de México. Revista Internacional de Estadísticas y Geografía, 4(1), 7289.

Secretaría del Medio Ambiente de Ciudad de México [Sedema]. (2015). Reporte de plantas de tratamiento de aguas residuales Ciudad de México. Sedema. 
Superintendencia de Servicios Públicos Domiciliarios [Superservicios]. (2017). Estudio Sectorial de los servicios públicos domiciliarios de Acueducto y Alcantarillado. Superservicios.

Superintendencia Nacional de Servicios de Saneamiento de Perú [sunAss]. (2015). Diagnóstico situacional de los sistemas de tratamiento de aguas residuales en las EPS del Perú y propuestas de solución. Perú.

Superintendencia Nacional de Servicios de Saneamiento de Perú [SUNASS]. (2017). Diagnóstico de las plantas de tratamiento de aguas residuales en el ámbito de operación de las entidades prestadoras de servicios de saneamiento. SUNASS.

Universidad Santo Tomás [USTA]. (2017). Municipios de la cuenca del río Bogotá siguen sin cumplir la norma. El Buscador, 3, 18. https://issuu. com/elbuscadorusta/docs/eb-03-2017_web. compressed

Vivid Economics (2015). Global greenhouse gas abatement opportunities from energy efficiency in wastewater treatment. Xylem Inc. 